\title{
The autokinesis of an after-image
}

\section{LEONARD BROSGOLE, ${ }^{1}$ St. John's University, Jamaica, N.Y. 11432}

An after-image was found to move autokinetically in the absence of voluntary eye movements. Its direction of motion coincided with that of a normally viewed stimulus. The apparent movement of such a stabilized image could not be explained in terms of local sign theory.

The eye movement interpretation of autokinesis received vigorous support from Matin \& Mackinnon (1964). They stabilized the image of an isolated visual target along a horizontal retinal meridian. However, the image was permitted to displace in a vertical direction. As a result of this procedure, lateral autokinesis was markedly reduced, while there was a pronounced increase vertically. Accordingly, the authors concluded that such apparent motion is contingent upon the retinal displacement of a target, produced by unmonitored shifts in eye position.

It would follow logically that an image stabilized vertically, as well as horizontally, could not possibly be seen to move in the absence of volitional eye movements. Therefore, the purpose of the following study was to determine whether or not an after-image can undergo autokinesis under conditions of eye fixation.

\section{METHOD}

This study was designed to assess the direction and extent of autokinesis with both stabilized and normal viewing. Image stabilization was accomplished by generating a negative afterimage with a strobe flash. The transient nature of the negative after-image initially posed a major problem for this experiment. According to preliminary findings, the after-image tended to dissipate during the latency period normally required for autokinesis. That is to say, whereas the after-image vanished within 7-15 sec, from 12-90 sec had to elapse before a normally viewed target, of a comparably judged size and brightness, appeared to move. Therefore, the logic of the stabilization condition centered about permitting the latency period to expire before producing the after-image. An appreciation of this problem should add some clarity to the apparatus and procedure to be described.

Apparatus

The apparatus consisted of a 1-in. diameter, circular patch of Sylvania, white, electroluminescent Tape-Lite with a $1 / 8$ in. diameter aperture in its center. A small red pilot light was secured directly behind the aperture, thus serving as a fixation target. The Tape-Lite was surrounded by the reflector of a flash unit attached to a Lafayette Instrument, E300, electronic stroboscope.

The apparatus was located $10 \mathrm{in.}$ in front of a wall which contained a grid, dividing it into an $x-y$ coordinate system. The fixation target was aligned with the intersection of the $x$ and $y$ axes.

Procedure

The experiment, consisting of two conditions, took place in total darkness. $S$ sat 46 in. from the apparatus with his head fixed in a Bausch and Lomb chin rest. The chin rest was adjusted so that the red fixation point was at eye level, as well as straight ahead. $S$ wore a pair of standard safety goggles containing a set of $1 / 4$ in. diameter artificial pupils. ${ }^{2}$

In the first, or stabilization condition, $S$ viewed the fixation point until it appeared to move. The reported autokinesis of the pilot light indicated that the latency period has elapsed. The stroboscope was then activated. The single flash, lasting for approximately $1 / 1000 \mathrm{sec}$, gave rise to a sequence of after-images. A brilliant, blue, negative after-image appeared about $2 \mathrm{sec}$ later. $\mathrm{S}$ then gazed directly at the fixation point, holding it in the center of the after-image.

The room lights were turned on after 5 sec of viewing, ${ }^{3}$ and $S$ indicated where the after-image had last appeared by placing the tip of a black board pointer upon the wall behind the apparatus. The apparent terminal position of the after-image was read directly off the grid. This procedure continued for 30 such trials. The first five were considered training trials and, therefore, were omitted in the analysis of the data.

The second condition was identical to the first, except that the Tape-Lite was turned on when S signaled the onset of autokinesis in the fixation point. Instead of the after-image, the Tape-Lite now served as the autokinetic stimulus to be viewed normally for 5 sec. $^{4}$ The procedure continued, just as before, for 30 trials-the first five constituting training trials. The order of the two conditions were counterbalanced across Ss.

Subjects

Six males and two females participated in this study. Ranging in age from 19-24, their mean age was 21.7 years. They were all psychology students who were naive as to the nature of this experiment.

\section{RESULTS AND DISCUSSION}

According to local signal theory, only a voluntary shift of the eye could give rise to the impression that an after-image is moving. Condition 1 was designed to prevent $S$ from volitionally pursuing the after-image, by requiring him to keep the fixation point centered within it. Since each $S$ allegedly accomplished this task, eye movements could be ruled out as a factor possibly initiating a sense of apparent motion. ${ }^{5}$

In Condition 1, autokinesis was reported in 190 of the 200 trials, or in $95 \%$ of the cases. The after-image appeared to drift through space with the fixation target located in its center. In other words, both were seen to move as a unit. Of the 190 instances of subjective motion, $58 \%$ had some northerly component, $25 \%$ were generally to the south, and $17 \%$ were purely lateral. Disregarding direction, the mean extent of autokinesis was $3.93 \mathrm{deg}$ of visual arc.

In Condition 2, autokinesis was seen $53 \%$ of the time, or in 105 of the 200 trials. Considering only those cases of subjective motion, $76 \%$ were primarily to the north, $16 \%$ had a downward trend, and $8 \%$ were purely east and west. lgnoring direction, 5.5 deg of autokinesis was seen. On 95 separate occasions ( $47 \%$ of the trials) the sudden appearance of the autokinetic stimulus resulted in a shift of the fixation target back to its point of origin, where it then remained perfectly stationary.

To integrate the findings, it would seem that a stabilized image is seen to move far more frequently than a normally viewed target, although to a somewhat lesser degree. Both conditions were comparable in that there was a predominance of apparent upward motion, followed by downward autokinesis, with strict horizontal movement being least preferred.

The intent of this study was not to compare the two conditions directly. The important point is that the autokinesis of an after-image is readily observed in the absence of voluntary eye movements. Condition 2 was of significance in that it showed that the apparent movement of a stabilized image demonstrates the same directional tendencies as a normally viewed stimulus. This obviously refutes an eye movement interpretation of autokinesis.

Therefore, those inferences derived from the findings of Matin and MacKinnon must be categorically rejected. Their conclusions reflect a failure to recognize the distinction between the initiation and allocation of visually perceived motion. Lateral stabilization merely increased S's propensity to see vertical movement, instead of radically reducing the frequency with which autokinesis was reported. Retinal displacement, then, seemed to affect the direction to which motion was attributed, as opposed to those antecedent factors which generate apparent motion.

Our data indicate that retinal displacement has no bearing upon the perception of motion.

\section{REFERENCES}

MATIN, L., \& MacKINNON, G. E. Autokinetic movement: Selective manipu- 
lation of directional components by image stabilization. Science, 1964, 143, 147-148.

\section{NOTES}

1. The author extends his appreciation to William Stoehr, Jr., for his role in gathering data and to Robert Zenhausern for his many helpful suggestions.

2. The artificial pupils served to occlude the extraneous light produced by the strobe flash in generating the after-image.

3. The $5 \mathrm{sec}$ viewing interval guaranteed that all $\mathrm{Ss}$ would have an equal a- mount of experience with the autokinetic phenomenon. It was feared that if a longer inspection period were used, some might tend to lose the afterimage.

4. The Tape-Lite stimulus was previously judged to be of equal size and brightness to the after-image when set at $2.3 \mathrm{ft}-\mathrm{L}$.

5. A change in eye position would result in a displacement of the fixation point relative to the after-image. The absence of such relative displacement indicated that the eyes were not moving. 